\title{
Synchronization in Coupled Sine Circle Maps
}

\author{
Nandini Chatterjee and Neelima Gupte \\ Department of Physics, University of Pune, Pune 411 007, India
}

\begin{abstract}
We study the spatially synchronized and temporally periodic solutions of a 1-d lattice of coupled sine circle maps. We carry out an analytic stability analysis of this spatially synchronized and temporally periodic case and obtain the stability matrix in a neat block diagonal form. We find spatially synchronized behaviour over a substantial range of parameter space. We have also extended the analysis to higher spatial periods with similar results. Numerical simulations for various temporal periods of the synchronized solution, reveal that the entire structure of the Arnold tongues and the devil's staircase seen in the case of the single circle map can also be observed for the synchronized coupled sine circle map lattice. Our formalism should be useful in the study of spatially periodic behaviour in other coupled map lattices.
\end{abstract}

PACS number(s) : 05.45. +b 
The study of complex dynamical behaviour in extended systems is currently of interest in a wide variety of contexts. The modelling and characterization of spatiotemporal behaviour in such systems [1,2] can provide insights into the complex behaviour found in diverse systems like oscillator arrays [3 8], coupled Josephson junction arrays [9], reaction diffusion systems 10,11], charge density waves [12], biological systems [13, 14, and turbulence in fluids [15]. The spatially extended nature of the system permits the appearance of complex spatio-temporal behaviour such as spatially periodic, quasi-periodic or chaotic behaviour with the concurrent appearance of temporally periodic, quasi-periodic or chaotic behaviour. An understanding of the rich variety of modes which can be excited due to the interplay between spatial and temporal behaviour may provide a clue to phenomena like pattern formation in natural systems [1, 16, 17] and turbulence.

Coupled Map Lattice (CML) models have recently attracted much attention in the study of spatio-temporal chaos and pattern formation as models of spatially extended systems [1, 2]. A CML is a dynamical system with discrete time, discrete space and continuous states. It consists of dynamical elements on a lattice which are suitably coupled. Such systems have been used succesfully to model real life phenomena like spiral waves [16] and spatio-temporal intermittency [18]. Though CML models are idealized systems, they are sufficiently complex to be capable of capturing the essential features of the dynamics of the system, and at the same time have the advantage of being mathematically tractable and computationally efficient.

Due to the large number of degrees of freedom in such spatially extended systems, a variety of spatio-temporal phenomena, like synchronization, intermittency, and spatio-temporal chaos are observed. One of the most important and interesting modes which can arise in such systems is the mode corresponding to synchronised behaviour, i.e. behaviour in which evolution at each spatial location is identical with that at every other spatial location at any arbitrary instant of time. (SeeFig.1a) Such synchronized behaviour has been observed in a number of spatially extended systems [19], like coupled oscillator arrays [5,9,20], coupled pendulums [21], electronic oscillator circuits 22] and in pattern formation [23]. As coupled sine circle map systems [2] can constitute models which capture many of the essential features of the behaviour of such systems, we address the problem of synchronized or spatially homogeneous solutions in a 1-dimensional array of coupled sine circle maps. The temporal evolution of these states may be periodic, quasi-periodic or chaotic in nature. The present paper concentrates on synchronized states that are temporally periodic in behaviour.

The single circle map 24,25, is represented by

$$
\theta_{t+1}=f\left(\theta_{t}\right)=\theta_{t}+\Omega-\frac{K}{(2 \pi)} \sin \left(2 \pi \theta_{t}\right) \quad \bmod 1
$$

where $\theta_{t}$ is an angle, at time $t$ which lies between 0 and $1, K$ is the nonlinearity parameter and $\Omega$ is the period of the system for $K=0$. This is one of the simplest representations of physical phenomena involving periodic motion. This simple dynamical system which exhibits a tendency to mode lock, as the parameter $K$ is increased, is particularly suitable for the description of resonances between periodic motion [24]. An extensive study by Bak et al [25], shows the presence of the Arnold tongues in the $\Omega-K$ space and complete mode-locking, namely the Devil's staircase at $K=1$. Since the single sine circle map exhibits this tendency to mode-lock, it is interesting to study whether an array of such sine circle maps on a lattice suitably coupled, would demonstrate such a mode-locking spatially. Synchronization is the simplest example of such a phenomenon and we focus our attention on the synchronized states which are temporally periodic in an array of such coupled circle maps.

The specific model under study, is a 1-dimensional coupled map lattice of sine-circle maps with nearest neighbour diffusive symmetric normalized coupling (also called future coupled laplacian coupling) and periodic boundary conditions, and is given by

$$
\begin{aligned}
\theta_{t+1}(i) & =(1-\epsilon) f\left(\theta_{t}(i)\right)+\frac{\epsilon}{2} f\left(\theta_{t}(i+1)\right)+\frac{\epsilon}{2} f\left(\theta_{t}(i-1)\right) \bmod 1 \\
& =(1-\epsilon)\left(\theta_{t}(i)+\Omega-\frac{K}{(2 \pi)} \sin \left(2 \pi \theta_{t}(i)\right)\right) \\
& +\frac{\epsilon}{2}\left\{\theta_{t}(i+1)+\Omega-\frac{K}{(2 \pi)} \sin \left(2 \pi \theta_{t}(i+1)\right)\right. \\
& \left.+\theta_{t}(i-1)+\Omega-\frac{K}{(2 \pi)} \sin \left(2 \pi \theta_{t}(i-1)\right)\right\} \bmod 1
\end{aligned}
$$

where $\theta_{t}(i)$ is the angular variable associated with the $i$ th site, at time $t$.

The parameters $\Omega$ and $K$ are taken to be uniform at each site and defined as in Eq.2 for the single circle map, and $\epsilon$ which lies between 0 and 1 is the strength of the coupling parameter.

We investigate in detail the phenomena of spatial synchronization in a system of such coupled sine circle maps. We identify the spatially homogeneous and temporally periodic, quasi-periodic and chaotic modes of the system. 
We carry out a linear stability analysis to analyse the stability properties of the spatially synchronized, temporally periodic solutions. The independent variables of the problem are identified and the analysis is cast in terms of these independent variables. This leads to a neat and simple block diagonal form for the stability matrix. This permits us to identify the regions in parameter space where synchronized solutions of different temporal periods are stable. The limits of the mode-locked interval for the temporal period one case can be explicitly evaluated due to the fact that the stability matrix has a block diagonal, block circulant structure, for this case. This analysis is also extended to higher spatial periods. Our method is quite general and should prove to be useful in the study of spatially periodic behaviour in other coupled map lattice models as well.

The paper is divided into five sections. Section I discusses the coupled shift map, which is the linear version of the coupled circle map, namely $K=0$ in Eq .2, and is thus the simplest case. We identify the independent variables for this system and recast the equation of evolution in terms of these variables. We carry out a systematic linear stability analysis, and obtain a neat form for the stability matrix. In section II, we extend the formalism to the system of coupled sine circle maps, taking into account the nonlinear sine term as defined in Eq. 2 and cast the problem in terms of the new independent variables of this system. The linear stability analysis in terms of these variables, gives a neat block diagonal form for the stability matrix. This is followed by an explicit calculation of the eigenvalues for the synchronized solution and the evaluation of the limits of the mode-locked interval for the fixed point case. Section III presents the generalization to higher spatial periods. In section IV, the numerical simulations for the higher temporal periods, of the synchronized solution, are discussed. We extend the algorithm developed by Bak et al [25], for the single circle map, to the synchronized solution of a system of coupled sine circle maps.Section V concludes with results and a short discussion.

\section{ANALYSIS FOR THE SHIFT MAP LATTICE}

We begin by carrying out the analysis for the simplest case of Eq. 2, namely for $K=0$, which is the case of coupled shift maps.

The single shift map i.e., the linear case of the single circle map, namely $K=0$ in Eq.1 is given by

$$
\theta_{t+1}=\theta_{t}+\Omega \quad \bmod 1
$$

This has periodic orbits for rational values of $\Omega$ i. e. $\Omega=\frac{P}{Q}$ where $P$ and $Q$ are any integers, and quasi-periodic orbits for irrational values of $\Omega$.

Eq. 2, with $K=0$, for the coupled shift map, for a lattice of $N$ sites, retaining periodic boundary conditions, reduces to

$$
\theta_{t+1}(i)=(1-\epsilon)\left(\theta_{t}(i)+\Omega\right)+\frac{\epsilon}{2}\left(\theta_{t}(i+1)+\theta_{t}(i-1)\right)+2 \Omega \quad \bmod 1
$$

For the synchronized solution, since the value of the variable at all sites is the same, we note that differences of nearest neighbour variable site values is equal to zero for all neighbours. Writing an equation for such a difference for the first pair of sites of an array of $N$ sites, for the coupled shift map, we obtain,

$$
\begin{aligned}
\theta_{t+1}(1)-\theta_{t+1}(2) & =(1-\epsilon)\left(\theta_{t}(1)-\theta_{t}(2)\right) \\
& +\frac{\epsilon}{2}\left(\theta_{t}(2)-\theta_{t}(3)+\theta_{t}(1)-\theta_{t}(N)\right)
\end{aligned}
$$

It is clear that Eq.5 can be expressed completely in terms of the differences defined by

$$
a_{t}(i)=\theta_{t}(i)-\theta_{t}(i+1)
$$

The evolution equation of the differences is given by,

$$
a_{t+1}(i)=(1-\epsilon)\left(a_{t}(i)\right)+\frac{\epsilon}{2}\left(a_{t}(i+1)+a_{t}(i-1)\right) \quad \bmod 1
$$

Thus, for the synchronized solution, of the coupled shift map, it is sufficient to define just $N$ differences, to obtain the $N$ equations of evolution in terms of independent variables.

Substituting $a_{t}(i)=0$, in Eq.7, gives $a_{t+1}(i)=0$, and hence shows that $a_{t}(i)=0$, is a spatial fixed point solution.

Expanding up to the linear term about this solution leads to a stability matrix $J_{t}^{\text {shift }}$, given by 


$$
J_{t}^{\text {shift }}=\left(\begin{array}{cccccccc}
(1-\epsilon) & \frac{\epsilon}{2} & 0 & 0 & \cdots & 0 & 0 & \frac{\epsilon}{2} \\
\frac{\epsilon}{2} & (1-\epsilon) & \frac{\epsilon}{2} & 0 & 0 & \cdots & 0 & 0 \\
0 & \frac{\epsilon}{2} & (1-\epsilon) & \frac{\epsilon}{2} & 0 & \cdots & 0 & 0 \\
\vdots & \vdots & \vdots & \vdots & \vdots & \vdots & \vdots & \vdots \\
\frac{\epsilon}{2} & 0 & 0 & \cdots & 0 & 0 & \frac{\epsilon}{2} & (1-\epsilon)
\end{array}\right)
$$

This is an $N \times N$ matrix, which is also circulant and whose eigen values maybe explicitly obtained analytically.

The eigenvalues of $J_{t}^{\text {shift }}$ are given by [26,27]

$$
\lambda_{r}=(1-\epsilon)+\frac{\epsilon}{2}\left(\omega_{r}+\omega_{r}^{N-1}\right)
$$

where $\omega_{r}$ is the $N$ th root of unity given by

$$
\omega_{r}=\exp \left(\frac{2 i \pi(r-1)}{N}\right)
$$

On simplifying, this can be written as

$$
\lambda_{r}=(1-\epsilon)+\epsilon \cos \left(\frac{2 \pi(r-1)}{N}\right) r=1,2, \ldots, N
$$

The largest eigenvalue of Eq. 11 namely $\lambda^{\text {largest }} \leq 1$ defines the stability condition for temporally stable and spatially synchronised periodic orbits of the coupled shift map. The largest eigen value is +1 , indicating that the coupled shift map is marginally stable. We find that as long as the differences between nearest neighbour sites tend to zero, we obtain synchronized solutions, irrespective of the corresponding temporal period. Since, the largest eigen value does not depend on $\epsilon$, we conclude that, for the synchronized solution, there is no dependence of the coupling parameter on the temporal behaviour. Thus for the synchronized solution, the coupled shift map continues to have periodic orbits for rational values of $\Omega$, and quasi-periodic orbits for $\Omega$ irrational, similar to the single shift map and there is no enlargement in the phase space due to the coupling.

\section{LINEAR STABILITY ANALYSIS FOR THE COUPLED CIRCLE MAP LATTICE}

We now carry out a similar linear stability analysis with the non-linear terms i.e. for the coupled circle maps as defined in Eq.2 with $K \neq 0$. We consider a 1-d closed chain of $N$ lattice sites, with the sine circle map at each site, coupled to its nearest neighbours via diffusive normalized symmetric coupling and periodic boundary conditions, as in Eq.2, such that the right hand neighbour of the $N$ th point is the first lattice point.

As discussed in the case of the coupled shift maps, for a synchronised solution, it makes sense to consider the evolution of the differences. At any arbitrary time $t$ we write an equation similar to Eq.5, now for the coupled sine circle map. Consider, as an example, the first pair of lattice sites, of a lattice of $\mathrm{N}$ sites, with evolution at each site defined as in Eq.2.

$$
\begin{aligned}
\theta_{t+1}(1)-\theta_{t+1}(2) & =(1-\epsilon)\left\{\left(\theta_{t}(1)-\theta_{t}(2)\right)\right. \\
& \left.-\frac{K}{\pi} \sin \left(\pi\left(\theta_{t}(1)-\theta_{t}(2)\right)\right) \cos \left(\pi\left(\theta_{t}(1)+\theta_{t}(2)\right)\right)\right\} \\
& +\frac{\epsilon}{2}\left\{\left(\theta_{t}(1)-\theta_{t}(N)\right)+\left(\theta_{t}(2)-\theta_{t}(3)\right)\right. \\
& -\frac{K}{\pi} \sin \left(\pi\left(\theta_{t}(1)-\theta_{t}(N)\right)\right) \cos \left(\pi\left(\theta_{t}(1)+\theta_{t}(N)\right)\right) \\
& \left.-\frac{K}{\pi} \sin \left(\pi\left(\theta_{t}(2)-\theta_{t}(3)\right)\right) \cos \left(\pi\left(\theta_{t}(2)+\theta_{t}(3)\right)\right)\right\} \bmod 1
\end{aligned}
$$

Eq.12 shows, that for the coupled sine circle map, the evolution equation for differences between the variable values of the nearest neighbours involves not just the differences between pairs of neighbours but also the sum of the variable values of the nearest neighbours, unlike the coupled shift map where the evolution equation could be defined in terms 
of the differences alone. However, it is interesting to note that for a synchronised solution, the difference of nearest neighbour lattice sites is zero, and the sum of nearest neighbour lattice sites is a constant, for a fixed $\Omega$ and $K$. Therefore, we write a second equation for the sums of the variables at nearest neighbour sites, and obtain,

$$
\begin{aligned}
\theta_{t+1}(1)+\theta_{t+1}(2) & =(1-\epsilon)\left\{\left(\theta_{t}(1)+\theta_{t}(2)\right)\right. \\
& \left.-\frac{K}{\pi} \sin \left(\pi\left(\theta_{t}(1)+\theta_{t}(2)\right)\right) \cos \left(\pi\left(\theta_{t}(1)-\theta_{t}(2)\right)\right)\right\} \\
& +\frac{\epsilon}{2}\left\{\left(\theta_{t}(1)+\theta_{t}(N)\right)+\left(\theta_{t}(2)+\theta_{t}(3)\right)\right. \\
& -\frac{K}{\pi} \sin \left(\pi\left(\theta_{t}(1)+\theta_{t}(N)\right)\right) \cos \left(\pi\left(\theta_{t}(1)-\theta_{t}(N)\right)\right) \\
& \left.-\frac{K}{\pi} \sin \left(\pi\left(\theta_{t}(2)+\theta_{t}(3)\right)\right) \cos \left(\pi\left(\theta_{t}(2)-\theta_{t}(3)\right)\right)\right\}+2 \Omega \bmod 1
\end{aligned}
$$

which again involves both the sums and the differences of nearest neighbours. It is clear that for the synchronized solution of a lattice of coupled sine circle maps, we now need to define $N$ equations for the evolution of differences of nearest neighbour sites and $N$ equations for the evolution of sums of nearest neighbour sites as compared to $N$ differences alone for the coupled shift map. This is due to the fact that the shift map is a linear version of the circle map and in the case of coupled circle maps the non-linear sine term requires the identification of the second independent variable. At any time $t$, we define the differences and sums, of the nearest neighbours, as follows,

$$
\begin{aligned}
& a_{t}(i)=\theta_{t}(i)-\theta_{t}(i+1) \\
& b_{t}(i)=\theta_{t}(i)+\theta_{t}(i+1)
\end{aligned}
$$

Using Eq.2, we find that that $a_{t}(i)$ and $b_{t}(i)$ evolve via the following Eqs.

$$
\begin{aligned}
a_{t+1}(i) & =(1-\epsilon)\left(a_{t}(i)-\frac{K}{\pi} \sin \left(\pi a_{t}(i)\right) \cos \left(\pi b_{t}(i) i\right)\right) \\
& +\frac{\epsilon}{2}\left(a_{t}(i+1)-\frac{K}{\pi} \sin \left(\pi a_{t}(i+1)\right) \cos \left(\pi b_{t}(i+1)\right)\right) \\
& +\frac{\epsilon}{2}\left(a_{t}(i-1)-\frac{K}{\pi} \sin \left(\pi a_{t}(i-1)\right) \cos \left(\pi b_{t}(i-1)\right) \quad \bmod 1\right.
\end{aligned}
$$

and

$$
\begin{aligned}
b_{t+1}(i) & =(1-\epsilon)\left(b_{t}(i)-\frac{K}{\pi} \sin \left(\pi b_{t}(i)\right) \cos \left(\pi a_{t}(i)\right)\right) \\
& +\frac{\epsilon}{2}\left(b_{t}(i+1)-\frac{K}{\pi} \sin \left(\pi b_{t}(i+1)\right) \cos \left(\pi a_{t}(i+1)\right)\right) \\
& +\frac{\epsilon}{2}\left(b_{t}(i-1)-\frac{K}{\pi} \sin \left(\pi b_{t}(i-1)\right) \cos \left(\pi a_{t}(i-1)\right)\right)+2 \Omega \bmod 1
\end{aligned}
$$

For a synchronized solution, as mentioned above, at any time $t$,

$$
\begin{aligned}
& a_{t}(i)=0 \\
& b_{t}(i)=\text { constant }=s
\end{aligned}
$$

Inserting these conditions, Eq.16 reduces to

$$
a_{t+1}(i)=0
$$

implying that $a_{t}(i)=0$ is a spatial fixed point solution.

Similarly, Eq.17 reduces to 


$$
b_{t+1}(i)=(1-\epsilon)\left(s-\frac{K}{\pi} \sin (\pi s)\right)+\epsilon\left(s-\frac{K}{\pi} \sin (\pi s)\right)+2 \Omega \quad \bmod 1
$$

which is again a constant (not necessarily the same constant) for a fixed $\Omega$ and $K$.

Eq.21 is not restricted to temporal evolution of any particular kind, implying that this same equation may be used to study temporally periodic, quasiperiodic and chaotic behaviour. As an example, $b_{t+1}(i)=$ the same constants will indicate a temporal fixed point solution and so on.

Thus $a_{t}(i)=0$ and $b_{t}(i)=$ constant are solutions of the equations of evolution. We now perform a Taylor expansion up to first order about these solutions to obtain the linear stability matrix $J_{t}$ which is of order $2 N \times 2 N$ and of the form

$$
J_{t}=\left(\begin{array}{cc}
A_{t}^{\prime} & B_{t}^{\prime} \\
B_{t}^{\prime} & A_{t}^{\prime}
\end{array}\right)
$$

where

$$
A_{t}^{\prime}=\left(\begin{array}{cccccc}
\epsilon_{s} A_{t}(1) & \epsilon_{n} A_{t}(2) & 0 & \cdots & 0 & \epsilon_{n} A_{t}(N) \\
\epsilon_{n} A_{t}(1) & \epsilon_{s} A_{t}(2) & \epsilon_{n} A_{t}(3) & 0 & \cdots & 0 \\
0 & \epsilon_{n} A_{t}(2) & \epsilon_{s} A_{t}(3) & \cdots & 0 & 0 \\
\vdots & \vdots & \vdots & \vdots & \vdots & \vdots \\
\epsilon_{n} A_{t}(1) & 0 & \cdots & 0 & \epsilon_{n} A_{t}(N-1) & \epsilon_{s} A_{t}(N)
\end{array}\right)
$$

and

$$
B_{t}^{\prime}=\left(\begin{array}{cccccc}
\epsilon_{s} B_{t}(1) & \epsilon_{n} B_{t}(2) & 0 & \ldots & 0 & \epsilon_{n} B_{t}(N) \\
\epsilon_{n} B_{t}(1) & \epsilon_{s} B_{t}(2) & \epsilon_{n} B_{t}(3) & 0 & \cdots & 0 \\
0 & \epsilon_{n} B_{t}(2) & \epsilon_{s} B_{t}(3) & \cdots & 0 & 0 \\
\vdots & \vdots & \vdots & \vdots & \vdots & \vdots \\
\epsilon_{n} B_{t}(1) & 0 & \cdots & 0 & \epsilon_{n} B_{t}(N-1) & \epsilon_{s} B_{t}(N)
\end{array}\right)
$$

Here

$$
\epsilon_{s}=(1-\epsilon), \quad \epsilon_{n}=\frac{\epsilon}{2}
$$

Each

$$
A_{t}(i)=\left(1-\frac{K}{\pi} \cos \left(\pi a_{t}(i)\right) \cos \left(\pi b_{t}(i)\right)\right)
$$

and

$$
B_{t}(i)=\left(1-\frac{K}{\pi} \sin \left(\pi a_{t}(i)\right) \sin \left(\pi b_{t}(i)\right)\right)
$$

Imposing the condition $a_{t}(i)=0, b_{t}(i)=s$ the stability matrix $J_{t}$, Eq.22 reduces to

$$
J_{t}=\left(\begin{array}{cc}
M_{t} & 0 \\
0 & M_{t}
\end{array}\right)
$$

where each $M_{t}$ is an $N \times N$ circulant matrix given by,

$$
M_{t}=\left(\begin{array}{cccc}
\epsilon_{s} \bar{A}_{t}(1) & \epsilon_{n} \bar{A}_{t}(2) & \cdots & \epsilon_{n} \bar{A}_{t}(N) \\
\epsilon_{n} \bar{A}_{t}(1) & \epsilon_{s} \bar{A}_{t}(2) & \cdots & 0 \\
0 & \epsilon_{n} \bar{A}_{t}(2) & \cdots & 0 \\
\vdots & \vdots & \vdots & \vdots \\
\epsilon_{n} \bar{A}_{t}(1) & 0 & \cdots & \epsilon_{s} \bar{A}_{t}(N)
\end{array}\right)
$$

For a synchronised solution all the lattice sites have the same value and hence each $b_{t}(i)=s \forall i, 1, \ldots, N$. Thus each

$$
\bar{A}_{t}(i)=\left(1-\frac{K}{\pi} \cos (\pi s)\right)
$$


We thus have a block diagonal matrix with circulant blocks. In the stability analysis for other coupled map lattices carried out so far, it was necessary to find a similarity transformation to obtain the stability matrix in block diagonal form [26]. In this case, we no longer have to find such a transformation, but directly obtain the stability matrix in a block diagonal form.

To study the stability of the homogeneous solution, it is sufficient to obtain the eigenvalues of one of the blocks $M_{t}$.

The eigenvalues of $M_{t}$ are given by Eq.9 [26,27, but are now of the form

$$
\begin{aligned}
\lambda_{r} & =(1-\epsilon)(1-K \cos (\pi s)) \\
& +\omega_{r}\left(\frac{\epsilon}{2}(1-K \cos (\pi s))\right)+\omega_{r}^{N-1}\left(\frac{\epsilon}{2}(1-K \cos (\pi s))\right)
\end{aligned}
$$

where $\omega_{r}$ is defined as in Eq.10

On simplifying, Eq.31 can be written as

$$
\begin{gathered}
\lambda_{r}=(1-\epsilon)(1-K \cos (\pi s))+\epsilon(1-K \cos (\pi s)) \cos \left(\frac{2 \pi(r-1)}{N}\right), \\
r=1,2, \ldots, N
\end{gathered}
$$

Now, $\left|\lambda_{r}\right|<1$, for all $\mathrm{r}$, ensures the stability of the synchronized solution. The largest eigenvalue $\lambda^{\text {largest }}$ crossing 1 , is the condition for marginal stability $[26]$.

As mentioned earlier, we have not assumed any temporal periodicity while defining $a_{t}(i)$ and $b_{t}(i)$. They have been defined at any arbitrary time $t$. Thus, as long as the condition for synchronization i.e. $a_{t}(i)=0$ and $b_{t}(i)=$ constant is satisfied, this formalism may be used to identify and model any kind of synchronised temporal behavior.

For the synchronized solution, we can analytically obtain those regions in the $\Omega-K-\epsilon$ space, for which the spatially synchronized (i.e. spatial period 1), and additionally temporal period 1 solution, is stable.

\section{A. The Fixed Point case}

For the temporal fixed point, i.e. $a_{t+1}(i)=a_{t}(i)=0$ and $b_{t+1}(i)=b_{t}(i)=s$, is also a synchronised solution and hence also the spatial fixed point of the system. Using Eq.32, the largest eigenvalue of the stability matrix $J_{t}$ is given by

$$
\lambda^{\text {largest }}=(1-K \cos (\pi s))
$$

Now, using the fixed point condition for Eq.(21),

$$
s=s-\frac{K}{\pi} \sin (\pi s)+2 \Omega
$$

This gives

$$
s=\frac{1}{\pi} \arcsin \left(\frac{2 \pi \Omega}{K}\right)
$$

Inserting this value of $s$ and using the condition for stability, $\lambda \pm 1$, in Eq. 33, we obtain the width of the stability interval,

$$
\Delta\left(\Omega\left(\frac{0}{1}\right)\right)=\left(\frac{-K}{2 \pi}, \frac{K}{2 \pi}\right)
$$

This is the same width, as that obtained by Bak et al [25] for the single circle map. This width is the width of that region of $\Omega$, for which we obtain stable fixed point solutions. Thus, we find that for the synchronized solution, the width of the $\frac{0}{1}$ interval for the coupled circle map case is identical to that obtained for the single circle map [25]. However, in this case, it is the width of the of the $\frac{0}{1}$ interval for each site, and since the value of $\Omega$ at each site is the same, for the entire lattice. Thus, for the region of $\Omega$, defined by Eq.36, the entire lattice, has synchronized stable temporal fixed point solutions.

For higher temporal periods, the stability matrix $J_{t}$, can be obtained in the following way. For a period $Q$ orbit, $J_{t}$ is simply given by, 


$$
J_{t}=\prod_{t=1}^{Q}\left(\begin{array}{ll}
A_{t}^{\prime} & B_{t}^{\prime} \\
B_{t}^{\prime} & A_{t}^{\prime}
\end{array}\right)
$$

where all the notation is the same as defined earlier.

Imposing, the condition for a synchronized solution, namely, Eqs.18 and 19, $J_{t}$ reduces to the following form

$$
J_{t}=\prod_{t=1}^{Q}\left(\begin{array}{cc}
M_{t} & 0 \\
0 & M_{t}
\end{array}\right)
$$

The widths of the the higher temporal periods, for the synchronized case cannot be obtained analytically,as has previously been observed in the case of the single circle map [25]. To calculate the widths of the higher temporal periods, for the synchronized solution we carry out numerical simulations which have been discussed in section IV. A detailed discussion is presented in [28].

Since the formalism set up here is sufficiently general, it may also be used to model synchronisation in other coupled map systems.

The next section extends the formalism for solutions corresponding to arbitrary spatial periods.

\section{ANALYSIS FOR THE $K$ TH SPATIAL PERIOD}

We find that the formalism discussed above may be efficiently used to study the stability of higher spatial periods as well. We extend it to higher spatial periods with the stability matrix retaining its block diagonal form. By higher spatial periods, say spatial period 4 , we mean every $i$ th and $(i+4)$ th lattice site will have the same value. (See Fig.1b)

We now extend this formalism, to an arbitrary spatial period $k$. At any time $t$, for a spatial period $k$ solution, the value of the variable at the $i$ th site is the same as the value at the $(i+k)$ th site. Thus, the difference, between the variable values of the $i$ th and the $(i+k)$ th site, approaches zero, while the sum of these variable values, approaches a constant. As discussed in section II, now for a spatial period $k$, we can show that the differences and sums, are again the independent variables of the system and are now defined as,

$$
\begin{aligned}
& a_{t}^{k}(i)=\theta_{t}(i)-\theta_{t}(i+k) \\
& b_{t}^{k}(i)=\theta_{t}(i)+\theta_{t}(i+k)
\end{aligned}
$$

where the superscript $k$ denotes the spatial period at any time $t$.

Eqs.16 and 17, now for a spatial period $k$, are modified to,

$$
\begin{aligned}
a_{t+1}^{k}(i) & =(1-\epsilon)\left(a_{t}^{k}(i)-\frac{K}{\pi} \sin \left(\pi a_{t}^{k}(i)\right) \cos \left(\pi b_{t}^{k}(i)\right)\right) \\
& +\frac{\epsilon}{2}\left(a_{t}^{k}(i+1)-\frac{K}{\pi} \sin \left(\pi a_{t}^{k}(i+1)\right) \cos \left(\pi b_{t}^{k}(i+1)\right)\right) \\
& +\frac{\epsilon}{2}\left(a_{t}^{k}(i-1)-\frac{K}{\pi} \sin \left(\pi a_{t}^{k}(i-1)\right) \cos \left(\pi b_{t}^{k}(i-1)\right)\right) \quad \bmod 1
\end{aligned}
$$

and

$$
\begin{aligned}
b_{t+1}^{k}(i) & =(1-\epsilon)\left(b_{t}(i)-\frac{K}{\pi} \sin \left(\pi b_{t}^{k}(i)\right) \cos \left(\pi a_{t}^{k}(i)\right)\right) \\
& +\frac{\epsilon}{2}\left(b_{t}^{k}(i+1)-\frac{K}{\pi} \sin \left(\pi b_{t}^{k}(i+1)\right) \cos \left(\pi a_{t}^{k}(i+1)\right)\right) \\
& +\frac{\epsilon}{2}\left(b_{t}^{k}(i-1)-\frac{K}{\pi} \sin \left(\pi b_{t}^{k}(i-1)\right) \cos \left(\pi a_{t}^{k}(i-1)\right)\right)+2 \Omega \bmod 1
\end{aligned}
$$

As shown in section II, here too it can be easily shown that, $\forall i, a_{t}^{k}(i)=0$ and $b_{t}^{k}(i)=s_{k}$, where $s_{1}, s_{2}, \ldots$ are all constants, and are solutions of the Eq. 41 and 42 for a fixed $\Omega$ and $K$. 

$J_{t}^{k}$

For a spatial period $k$ solution, we expand about, $a_{t}^{k}(i)=0$ and $b_{t}^{k}(i)=$ constant and obtain the stability matrix

$$
J_{t}^{k}=\left(\begin{array}{cc}
A_{t}^{\prime k} & B_{t}^{\prime k} \\
B_{t}^{\prime k} & A_{t}^{\prime k}
\end{array}\right)
$$

where

$$
A_{t}^{\prime k}=\left(\begin{array}{cccccc}
\epsilon_{s} A_{t}^{k}(1) & \epsilon_{n} A_{t}^{k}(2) & 0 & \ldots & 0 & \epsilon_{n} A_{t}^{k}(N) \\
\epsilon_{n} A_{t}^{k}(1) & \epsilon_{s} A_{t}^{k}(2) & \epsilon_{n} A_{t}^{k}(3) & 0 & \cdots & 0 \\
0 & \epsilon_{n} A_{t}^{k}(2) & \epsilon_{s} A_{t}^{k}(3) & \ldots & 0 & 0 \\
\vdots & \vdots & \vdots & \vdots & \vdots & \vdots \\
\epsilon_{n} A_{t}^{k}(1) & 0 & \cdots & 0 & \epsilon_{n} A_{t}^{k}(N-1) & \epsilon_{s} A_{t}^{k}(N)
\end{array}\right)
$$

and

$$
B_{t}^{\prime k}=\left(\begin{array}{cccccc}
\epsilon_{s} B_{t}^{k}(1) & \epsilon_{n} B_{t}^{k}(2) & 0 & \ldots & 0 & \epsilon_{n} B_{t}^{k}(N) \\
\epsilon_{n} B_{t}^{k}(1) & \epsilon_{s} B_{t}^{k}(2) & \epsilon_{n} B_{t}^{k}(3) & 0 & \ldots & 0 \\
0 & \epsilon_{n} B_{t}^{k}(2) & \epsilon_{s} B_{t}^{k}(3) & \ldots & 0 & 0 \\
\vdots & \vdots & \vdots & \vdots & \vdots & \vdots \\
\epsilon_{n} B_{t}^{k}(1) & 0 & \cdots & 0 & \epsilon_{n} B_{t}^{k}(N-1) & \epsilon_{s} B_{t}^{k}(N)
\end{array}\right)
$$

where $\epsilon_{s}, \epsilon_{n}$ are defined by Eq.25 Here, each

$$
A_{t}(i)=\left(1-\frac{K}{\pi} \cos \left(\pi a_{t}^{k}(i)\right) \cos \left(\pi b_{t}^{k}(i)\right)\right)
$$

and

$$
B_{t}(i)=\left(1-\frac{K}{\pi} \sin \left(\pi a_{t}^{k}(i)\right) \sin \left(\pi b_{t}^{k}(i)\right)\right)
$$

Imposing the conditions $a_{t}^{k}(i)=0$ and $b_{t}^{k}(i)=s_{k}$, the stability matrix $J_{t}^{k}$ given by Eq.43 again reduces to a block diagonal form

$$
J_{t}^{k}=\left(\begin{array}{cc}
M_{t}^{k} & 0 \\
0 & M_{t}^{k}
\end{array}\right)
$$

where each $M_{t}^{k}$ is of the form,

$$
M_{t}^{k}=\left(\begin{array}{cccc}
\epsilon_{s} \bar{A}_{t}^{k}(1) & \epsilon_{n} \bar{A}_{t}^{k}(2) & \cdots & \epsilon_{n} \bar{A}_{t}^{k}(N) \\
\epsilon_{n} \bar{A}_{t}^{k}(1) & \epsilon_{n} \bar{A}_{t}^{k}(2) & \cdots & \\
0 & \epsilon_{n} \bar{A}_{t}^{k}(2) & \cdots & 0 \\
\vdots & \vdots & \vdots & \vdots \\
\epsilon_{n} \bar{A}_{t}^{k}(1) & 0 & \cdots & \epsilon_{s} \bar{A}_{t}^{k}(N)
\end{array}\right)
$$

and each

$$
\bar{A}_{t}^{k}(i)=\left(1-\frac{K}{\pi} \cos \left(\pi s_{m}\right)\right)
$$

where, each $m$ goes from $\quad 1, \ldots, k$ See Fig.1(b) for an illustration of spatial period 4.

Thus even for higher spatial periods, the stability matrix $J_{t}^{k}$ is in a block diagonal form, Eq.48. Finding the eigen values of the matrix $M_{t}^{k}$, and the largest eigen value crossing +1 , gives the region of stability of the period $k$ solution. 


\section{NUMERICAL SIMULATIONS}

We obtained the width of $\Delta\left(\Omega\left(\frac{0}{1}\right)\right)$, the temporal period 1 case, for the synchronized solution analytically in section II. The higher temporal periods, $\Delta \Omega(P / Q)$, for the synchronized solution, are obtained numerically.

The widths, $\Delta \Omega(P / Q)$, as also stated in section II, are those regions of $\Omega$, at $K=1$, for which, the entire lattice, will have temporally stable and spatially synchronized periodic solutions of time period $Q$.

We extend the algorithm developed by Bak et al [25], in the following manner.

For a lattice of $N$ sites we define the following vector notation,

$$
\vec{f}(\vec{\theta}, \vec{\Omega}) \rightarrow\left\{f_{1}(\vec{\theta}, \vec{\Omega}), f_{2}(\vec{\theta}, \vec{\Omega}), \ldots, f_{N}(\vec{\theta}, \vec{\Omega})\right\}
$$

where $i$ denotes the lattice index and each $f_{i}(\vec{\theta}, \vec{\Omega})$ is defined by Eq.2 Each $\theta$ is now a vector of the form

$$
(\vec{\theta}) \rightarrow\left\{\theta_{t}(1), \theta_{t}(2), \ldots, \theta_{t}(N)\right\}
$$

and the parameter, $\Omega$, also a vector, is represented as

$$
(\vec{\Omega}) \rightarrow\{\Omega(1), \Omega(2), \ldots, \Omega(N)\} \rightarrow\{\Omega, \Omega, \ldots, \Omega\}
$$

$\Omega$, in principle may have different values at each site, but in this case has the same value at each site.

For a 1-d array of coupled sine circle maps, i.e. a multi-dimensional case, the stability criterion is obtained by examining the eigen values of the following $N \times N$ matrix

$$
S_{t}=\prod_{t=1}^{Q}\left(\begin{array}{cccc}
\frac{\partial f_{1}}{\partial \theta_{t}(1)} & \frac{\partial f_{1}}{\partial \theta_{t}(2)} & \cdots & \frac{\partial f_{1}}{\partial \theta_{t}(N)} \\
\frac{\partial f_{2}}{\partial \theta_{t}(1)} & \frac{\partial f_{2}}{\partial \theta_{t}(2)} & \cdots & \frac{\partial f_{2}}{\partial \theta_{t}(N)} \\
\vdots & \vdots & \vdots & \vdots \\
\frac{\partial \dot{f}_{N}}{\partial \theta_{t}(1)} & \frac{\partial \dot{f}_{N}}{\partial \theta_{t}(2)} & \cdots & \frac{\partial \dot{f}_{N}}{\partial \theta_{t}(N)}
\end{array}\right)
$$

Let $\left\{\lambda_{i}\right\}$ be the set of eigenvalues of the matrix $S_{t}$. For a period $Q$ orbit to be stable the eigenvalues of the matrix $S_{t}<1$. The largest eigenvalue crossing 1 defines the marginal stability condition.

Thus for the higher order temporal periods, we seek the solution to the set of Eqs. 55 and 56,

$$
\vec{f}^{Q}(\vec{\theta}, \vec{\Omega})=(\vec{\theta})+\vec{P}
$$

the condition for closure, and

$$
\lambda^{\text {largest }}=1
$$

the condition for marginal stability

For a general $P / Q$ step, and a lattice of $N$ sites, we start with homogeneous initial conditions and perform the iterative technique based on the Newton - Raphson method.

We define

$$
\begin{aligned}
& \vec{g}_{1}(\vec{\theta}, \vec{\Omega})=\left\{g_{1}(1), g_{1}(2), \ldots, g_{1}(N)\right\} \\
& \vec{g}_{2}(\vec{\theta}, \vec{\Omega})=\left\{\frac{\partial f_{1}^{Q}}{\partial \theta_{t}(1)}, \frac{\partial f_{2}^{Q}}{\partial \theta_{t}(2)}, \ldots, \frac{\partial f_{N}^{Q}}{\partial \theta_{t}(N)}\right\}
\end{aligned}
$$

where

$$
\vec{g}_{1}(1)=f_{1}^{Q}(\vec{\theta}, \vec{\Omega})-(\theta(1))-\vec{P}
$$

and so on.

We further define,

$$
\vec{g}(\vec{\theta}, \vec{\Omega})=\left(\begin{array}{c}
\overrightarrow{g_{1}}(\vec{\theta}, \vec{\Omega}) \\
\overrightarrow{g_{2}}(\vec{\theta}, \vec{\Omega})
\end{array}\right)
$$


where now,

$$
\vec{g}_{2}(\vec{\theta}, \vec{\Omega})=\vec{g}_{2}(\vec{\theta}, \vec{\Omega})-\overrightarrow{1}
$$

For the synchronized solution, it can be shown, that the largest eigenvalue of the matrix, $S_{t}$, namely $\lambda^{\text {largest }}$, is equal to each of the $N$ components of $\vec{g}_{2}$ in Eq.58 [28].

Therefore, Eqs.55 and 56, may now be expressed as finding $\left(\vec{\theta}^{*}, \vec{\Omega}^{*}\right)$ such that

$$
\vec{g}\left(\vec{\theta}^{*}, \vec{\Omega}^{*}\right)=\vec{g}^{*}=\overrightarrow{0}
$$

Taylor expanding $\vec{g}^{*}$, about the initial point of iteration, $\vec{g}\left(\vec{\theta}^{0}, \vec{\Omega}^{0}\right)=\vec{g}_{0}$, up to the linear order, we obtain,

$$
\vec{g} * \simeq \overrightarrow{g_{0}}+\vec{\Delta} M
$$

where

$$
\begin{gathered}
\vec{\Delta}=\left(\vec{\theta}^{*}, \vec{\Omega}^{*}\right)-\left(\vec{\theta}^{\mathrm{D}}, \vec{\Omega}^{0}\right) \\
M=\left(\begin{array}{cccccc}
\frac{\partial g_{1}(1)}{\partial \theta_{t}(1)} & \cdots & \frac{\partial g_{1}(1)}{\partial \theta_{t}(N)} & \frac{\partial g_{1}(1)}{\partial \Omega(1)} & \cdots & \frac{\partial g_{1}(1)}{\partial \Omega(N)} \\
\vdots & \vdots & \vdots & \vdots & \vdots & \vdots \\
\frac{\partial g_{1}(N)}{\partial \theta_{t}(1)} & \cdots & \frac{\partial g_{1}(N)}{\partial \theta_{t}(N)} & \frac{\partial g_{1}(N)}{\partial \Omega(1)} & \cdots & \frac{\partial g_{1}(N)}{\partial \Omega(N)} \\
\frac{\partial g_{2}(1)}{\partial \theta_{t}(1)} & \cdots & \frac{\partial g_{2}(1)}{\partial \theta_{t}(N)} & \frac{\partial g_{2}(1)}{\partial \Omega(1)} & \cdots & \frac{\partial g_{2}(1)}{\partial \Omega(N)} \\
\vdots & \vdots & \vdots & \vdots & \vdots & \vdots \\
\frac{\partial g_{2}(N)}{\partial \theta_{t}(1)} & \cdots & \frac{\partial g_{2}(N)}{\partial \theta_{t}(N)} & \frac{\partial g_{2}(N)}{\partial \Omega(1)} & \cdots & \frac{\partial g_{2}(N)}{\partial \Omega(N)}
\end{array}\right)
\end{gathered}
$$

It is found that, for

$$
\begin{gathered}
\vec{g}^{*}=\overrightarrow{0} \\
\vec{\Delta} \simeq-M^{-1} \vec{g}_{0}
\end{gathered}
$$

And so as a first approximation,

$$
\left(\begin{array}{c}
\vec{\theta}^{*} \\
\vec{\Omega}^{*}
\end{array}\right) \simeq\left(\begin{array}{c}
\vec{\theta}^{1} \\
\vec{\Omega}^{1}
\end{array}\right)=-M^{-1} \vec{g}_{0}+\left(\begin{array}{c}
\vec{\theta}^{0} \\
\vec{\Omega}^{0}
\end{array}\right)
$$

All derivatives can be derived recursively 28]

We start with homogeneous initial conditions, for the synchronized case, and find, to initiate the iteration, it is convenient to locate the superstable point $(\vec{\theta}, \vec{\Omega})=\left(\vec{\theta}_{s}, \vec{\Omega}_{s}\right)$, for all the lattice sites, where now the eigenvalues of the matrix $S_{t}$ are $=0$. All steps have been found to an accuracy of $10^{-8}$.

Using the alogrithm discussed above, at $K=1$ we obtain the complete devil's Staircase, of periodic orbits, now in the $\Omega-\frac{P}{Q}-\epsilon$ space (see Fig.2) with all the special features seen in the single circle map.

We also studied various features of the coupled sine circle map lattice at lower values of $K$ and different $\Omega$ 's. Our simulations reveal synchronized quasi-periodic orbits and periodic orbits in the $\Omega-K-\epsilon$ space. We thus obtain the entire structure of the Arnold tongues ( see Fig.3) now with the third coupling dimension $\epsilon$. It is clear from ( see Fig. 3 that the three possible routes to chaos seen in the case of the single circle map i.e. via mode-locking alone , via quasi-periodicity and mode-locking and via quasi-periodicity to chaos, will also be seen for the coupled sine circle map lattice.

Thus for the synchronized solution, we obtain all the features of the single map, now with a third dimension, in the form of the coupling parameter. We also observe that for the synchronised solution all the features as seen in the single circle map, are lifted exactly to this extra dimension in the parameter space, namely the coupling parameter $\epsilon$, for the entire range, $0 \leq \epsilon \leq 1$. 


\section{CONCLUSIONS}

We have set up a formalism to analyze the phenomenon of synchronization in a lattice of coupled sine circle maps. We identify the independent variables, the differences and the sums of variable values at neighbouring sites, $a_{t}(i)$ and $b_{t}(i)$ for the problem and show that, $a_{t}(i)=0$ and $b_{t}(i)=$ constant, the synchronised solution, are solutions, of the system under consideration. Casting the evolution equations in terms of these independent variables leads to a stability matrix which is in a neat block diagonal form. In addition, these blocks have a circulant structure, so we have a block diagonal matrix with circulant blocks. The temporal fixed point case, for the synchronised solution, i.e. the spatial period one and temporal period one solution can be solved explicitly to obtain the corresponding width of the stability interval. We have also demonstrated that this analysis can be easily extended to higher spatial periods. For an arbitrary spatial period $k$, we identify the independent variables, now $a_{t}^{k}(i)$ and $b_{t}^{k}(i)$ and again show that $a_{t}^{k}(i)=0$ and $b_{t}^{k}(i)=k$ constants, are solutions of the system. A linear stability analysis for this case shows that the stability matrix retains its block diagonal form. This analysis may also be easily extended to spatially periodic behaviour in other coupled map systems. The analysis is one of the simplest presented so far with the additional advantage of being transparent and neat.

We calculate the widths of the higher temporal periods for the synchronized case, by extending the algorithm developed by Baket al 25. Our numerical simulations reveal that the for the synchronised case, the entire structure of the Arnold Tongues and the devil's Staircase as observed for the single circle map is lifted to the chain of coupled sine circle maps.

A study of the parameter basins of attraction, i.e. those regions in the $\Omega-\epsilon$, where a particular class of initial conditions converges to the synchronized solution, which is the attractor, have also been carried out. These results which have been presented elsewhere [28] showed that these regions were found to be fairly large and showed an interesting symmetry about $\Omega=0.5$. Random initial conditions also revealed a finite basin of attraction, which was also symmetric about $\Omega=0.5$. The numerical simulations for the random initial conditions showed that there always exists a finite region of the $\Omega-\epsilon$ parameter space, at $K=1$, for which synchronized solutions will be obtained. The temporal period for these synchronized regions was found to be 1. [28].

Investigation of higher spatial periods reveals extremely interesting structures, results of which will be presented elsewhere. Our analysis was carried out for the homogenous case which assumed that the parameter values at each lattice site were the same. However the formalism set up is completely general and can be easily extended to inhomogeneous cases. This should be useful for the study of lattices where some lattice sites function as pinning sites.

We hope this work will find useful applications in the study of spatially periodic behaviour in systems like Josephson junction arrays and coupled oscillator arrays and in studies of pattern formation, in reaction-diffusion systems and charge-density waves.

\section{ACKNOWLEDGMENTS}

One of the authors (NG) acknowledges UGC and DST India, and (NC) gratefully acknowledges CSIR (India), for financial support. 


\section{References}

[1] K. Kaneko in Theory and Applications of Coupled Map Lattices, edited by K. Kaneko (John Wiley, England, 1993) and references therein.

[2] J. P. Crutchfield, and K. Kaneko in Directions in Chaos, edited by Hao-Bai Lin (World Scientific, Singapore, 1987), Vol 3 and references therein.

[3] M. Y. Choi et al, Phys.Rev. E 493825 (1994)

[4] G. L. Oopo and R. Kapral, Phys. Rev. A33, 4219 (1985); R. Kapral and G. L. Oopo, Physica 23D, 455 (1986).

[5] I. Waller and R. Kapral, Phys.Rev. 30A 2047 (1984); R. Kapral, Phys.Rev. 31A, 3868 (1985).

[6] T. Yamada and H. Fujisaka, Prog.Theo.Phys. 72885 (1985), 74918 (1985).

[7] R. van Buskirk and C. D. Jefferies, Phys.Rev. A 31, 3332 (1985).

[8] K. Kaneko, Physica D 34, 1 (1989).

[9] K. Wiesenfeld and P. Hadley, Phys. Rev. Lett. 62, 1335 (1989)

[10] R. Kapral in Theory and Applications of Coupled Map Lattices, edited by K. Kaneko (John Wiley, England, 1993) and references therein.

[11] F. Daviaud et al, Physica D 55, 287 (1992).

[12] J. Levy et al, Phys. Rev. Lett. 68, 2968 (1992).

[13] J. Nagumo and S. Salo, Kybernetik 10, 155 (1972).

[14] W. Freeman and C. A. Skarda, Brain Res. Rev 10147 (1985). W. Freeman, Brain Res. Rev. 11259 (1986).

[15] K. R. Sreenivasan, in Frontiers of Fluid Mechanics edited by J. J. Lumley (Springer-Verlag, Berlin, 1985) ; T. Schreiber, J. Phys. A 23, L393 (1990).

[16] D. Barkley, in Nonlinear Structures in Dynamical Systems, edited by Lui Lam and H. C. Morris (Springer - Verlag, New York 1991).

[17] H. L. Swinney and V. I. Krinsky in Waves and Patterns in Chemical and Biological Media, Physica 49D (1991). 50, 1874 (1994) and references therein.

[18] H. Chate and P. Mannevile, Physica D 32, 409 (1988).

[19] P. M. Gade, H. A. Cerdeira, R. Ramaswamy, to be published in Phys. Rev. E.

[20] J. F. Heagy, T. L. Carroll and L. M. Pecora Phys. Rev.E 50, 1874 (1994) and references therein

[21] F. F. Abraham, Phys. Rev. E 47, 1625 (1993).

[22] P. Ashwin et al., Nonlinearity 3, 585 (1990).

[23] K. Kaneko, Physica 34 D, 1-41 (1989).

[24] P. Bak, T. Bohr, and M. Jensen in Directions in Chaos, edited by Hao-Bai Lin (World Scientific, Singapore, 1987), Vol 2 and references therein.

[25] T. Bohr, P. Bak, M. H. Jensen, Phys.Rev. A 301960 (1984).

[26] P. M. Gade and R. E. Amritkar, Phys. Rev. E 47, 556 (1993).

[27] P. J. Davis, Circulant Matrices (Wiley, New York, 1979).

[28] N. Chatterjee and N. Gupte in, Proceedings of Dynamics of Complex Systems, 6th - 11th August, 1995, Calcutta, India, to be published by Physica $\mathbf{A}$. 


\section{FIGURE CAPTIONS}

FIG.1 (a) The synchronised or spatial period 1 solution

(b) The spatial period 4 solution

Each symbol in the figure represents a distinct value of the variable of a lattice of 8 sites at any time $t$.

FIG.2 The Devil Staircase for coupled sine circle maps.

The symmetry about $\Omega=0.5$ is clearly seen.

The widths are calculated for $\Delta \Omega\left(\frac{P}{Q}\right)$ for

$\frac{P}{Q}=\frac{0}{1}, \frac{1}{1}, \frac{1}{2}, \frac{1}{3}, \frac{2}{3}, \frac{1}{4}, \frac{3}{4}, \frac{1}{5}, \frac{2}{5}$.

This plot is for 8 lattice sites, but for a synchronized solution, identical results are seen for any $N$.

FIG.3 Plot of the Arnold tongues, for the coupled sine circle map lattice.

The tongues have been plotted for $\Omega=\frac{P}{Q}$ starting from

$\frac{P}{Q}=\frac{0}{1}, \frac{1}{1}, \frac{1}{2}, \frac{1}{3}, \frac{2}{3}$

This plot is for 8 lattice sites, but identical results are seen for any $N$, for the synchronized solution. 
Figure 1

a. $\bigcirc \quad \circ \quad \bigcirc \quad \circ \quad \circ \quad \bigcirc \quad 0 \quad 0$

b. $\square \circ 0+\square \circ 0+$ 
Figure 2

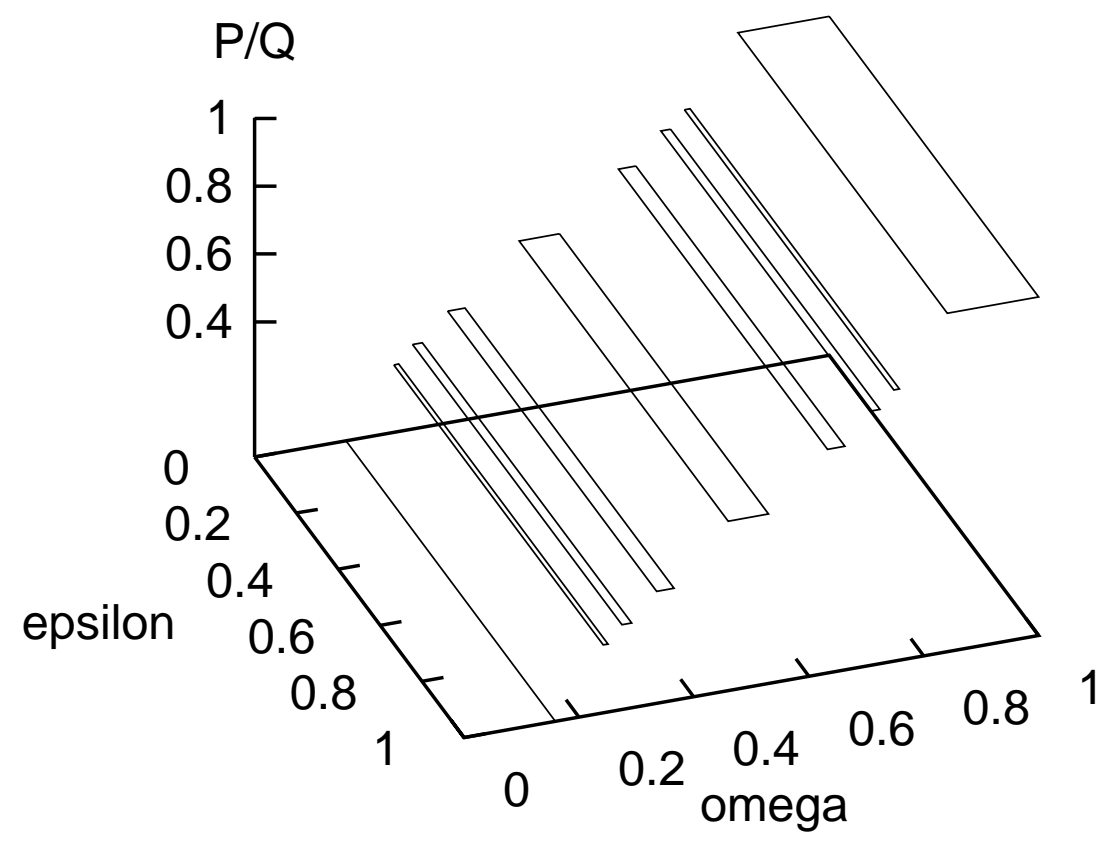


Figure 3

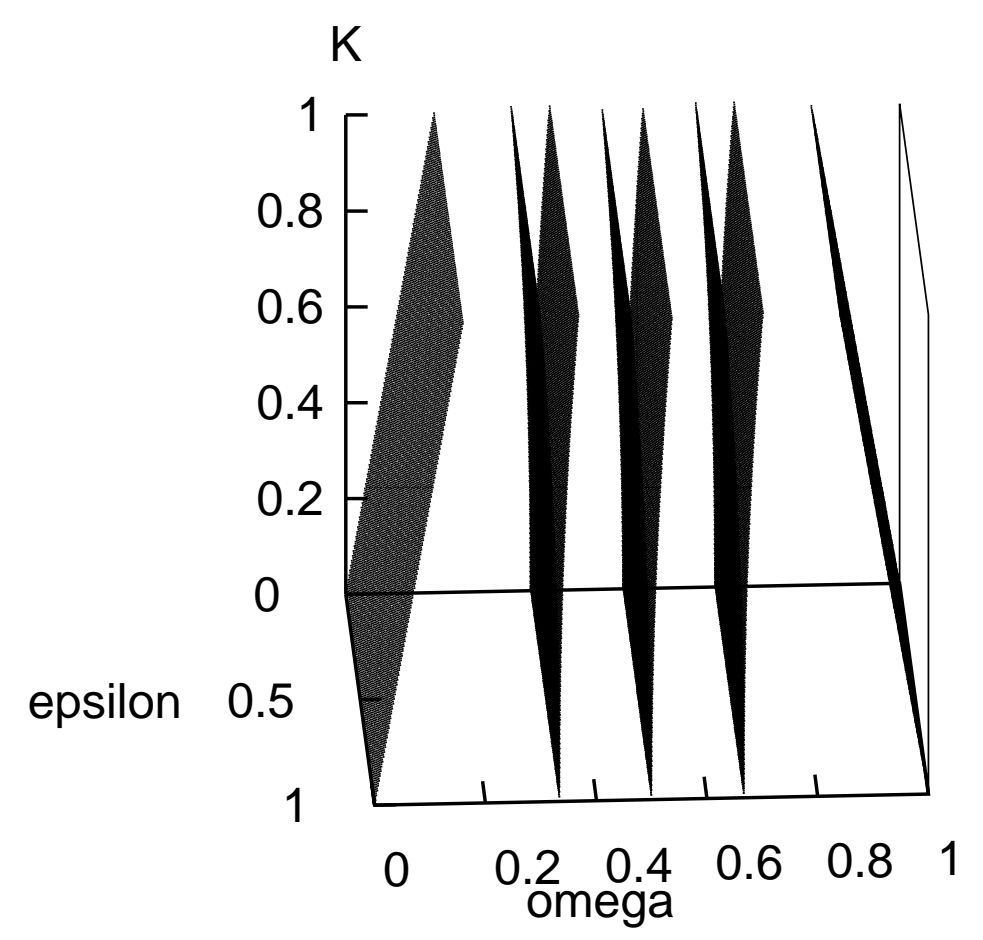

